Editorial

\title{
Seyfert Galaxies Astrophysics
}

\author{
Luigi Foschini \\ INAF, Brera Astronomical Observatory, 23807 Merate, Italy; luigi.foschini@inaf.it
}

Received: 10 August 2020; Accepted: 15 August 2020; Published: 17 August 2020

Keywords: active galactic nuclei; Seyfert galaxies; supermassive black hole; accretion physics; relativistic jets; winds and outflows; feedback; host galaxy

Looking back in time, it is somehow surprising to see the enormous advancement made by astrophysics in just about one century. At the dawn of the 20th century, the Universe was limited to the Milky Way and astronomers were debating about the nature of nebulae. To accomplish this task, Fath, Slipher, and Hubble observed these nebulae with spectroscopes. Among the many objects they observed, some displayed anomalously wide hydrogen emission lines. The first systematic study of these objects was done by Carl Seyfert in 1943 [1]. He studied six galaxies, today renowned: NGC 1068, NGC 1275, NGC 3516, NGC 4051, NGC 4151, and NGC 7469. He found high-excitation and prominent emission lines with full-width half maximum (FWHM) up to $\sim 8500 \mathrm{~km} \mathrm{~s}^{-1}$. He wrote that "The widths of the emission bands are probably correlated with the physical properties of the nucleus", but he did not suggest anything about these properties.

Today, we know that these anomalous widths are due to the presence of a supermassive black hole at the center of the galaxy. Its gravitational pull and the luminosity of the accretion disk around it significantly affect the nearby environment, thus determining the observational characteristics. Seyfert galaxies are available in many flavors: type 1 (face-on view), type 2 (edge-on view), intermediate, narrow-line Seyfert 1 (NLS1s, objects with smaller masses of the central black hole and highest accretion luminosity). Some researchers still set a distinction between the farthest and brightest, and the nearest and dimmest objects, according to their absolute optical blue magnitude $M_{\mathrm{B}}$ : quasi-stellar objects (QSO) and quasi-stellar radio objects (quasars) for the former, and simply Seyfert galaxies for the latter. However, as pointed out by Paola Marziani, Jack Sulentic, and Mauro D'Onofrio [2], today the divide of $M_{\mathrm{B}}=-23$ is just historical matter. The physical engine is the same and one should simply call them active galactic nuclei (AGN).

Although the topic offers a wide variety of possibilities, the contributions of the present Special Issue focus mainly on jetted NLS1s. The discovery in 2008 by Fermi/LAT of high-energy $\gamma$-ray emission from a handful of these objects [3-6] has renewed the interest in these AGN, after the Golden Age of ROSAT during the 1990s.

The first published contribution deals with $1 \mathrm{H} 0323+342(z=0.063)$, the nearest known $\gamma$-ray NLS1 [7]. Given its proximity, this object was studied in great detail, particularly at radio frequency, where it was possible to trace the relativistic jet as closer as possible to the central black hole. The article reviews many of the published works on $1 \mathrm{H} 0323+342$ aiming at building a map of its structure, from the smallest to the largest scale.

The second paper faces the problem to know how many NLS1s do have a relativistic jet [8]. Fan selected a sample of NLS1s from the Sloan Digital Sky Survey and cross-correlated it with the first release of LOFAR Two-meter Sky Survey (LoTSS DR1, 120-168 MHz). He found a detection rate of $28 \%$, decreasing to $12 \%$ and $1 \%$ when referring to the radio loudness values of 10 and 100 , respectively. A comparison sample of broad-line Seyferts resulted in rates of $36 \%, 8 \%$, and $1 \%$, respectively.

The third contribution is a study about the possibility of the forthcoming Cherenkov Telescope Array (CTA) to distinguish between different models of the high-energy $\gamma$-ray spectra $(E \gtrsim 20 \mathrm{GeV})[9]$. 
Vercellone proved that the superior performance of CTA will significantly increase the parameter space where it is possible to distinguish between a power-law and a log-parabola model, leaving the degeneracy restricted only to a very small range of unlikely values. If the log-parabola model will be confirmed by observations, there will be important consequences on the understanding of the mechanisms of particle acceleration.

The fourth article is a statistical analysis of a sample of jetted Seyfert galaxies selected at hard X-rays [10]. The authors measured and compared the observational characteristics of Seyfert type 1 and 2, discussing the differences and similarities. They suggest that there are different regimes of the jet-disk coupling depending on the Eddington ratio $\lambda$ : weak coupling for $\lambda<0.001$ and $0.3<\lambda<10$, strong and linear coupling in the middle range.

The last essay reports about an intriguing problem: the existence of obscured NLS1s [11]. In NLS1s, the narrowness refers to the broad permitted lines. The observation of the bump of FeII is a key factor in defining the NLS1, as it is a marker of the direct view of the broad-line region, which in turn means that the narrow lines are not from the narrow-line region. Pan studied three sources that may break this definition, as they might be NLS1s viewed at large angle, thus with (partial) obscuration along the viewing angle.

I would like to conclude this Editorial by thanking all the researchers who contributed to this Special Issue. Enjoy the reading!

Funding: This research received no external funding.

Conflicts of Interest: The author declares no conflict of interest.

\section{References}

1. Seyfert, C.K. Nuclear Emission in Spiral Nebulae. Astrophys. J. 1943, 97, 28-40. [CrossRef]

2. Marziani, P.; Sulentic, J.W.; D'Onofrio, M. An Introduction to 50 Years of Research on Quasars. In Fifty Years of Quasars; D'Onofrio, M., Marziani, P., Sulentic, J., Eds.; Springer: Berlin/Heidelberg, Germany, 2012; pp. 1-10.

3. Abdo, A.A.; et al. [Fermi LAT Collaboration] Fermi/Large Area Telescope discovery of gamma-ray emission from a relativistic jet in the narrow-line quasar PMN J0948 + 0022. Astrophys. J. 2009, 699, 976-984. [CrossRef]

4. Abdo, A.A.; et al. [Fermi LAT Collaboration] Multiwavelength monitoring of the enigmatic narrow-line Seyfert 1 PMN J0948+0022 in 2009 March-July. Astrophys. J. 2009, 707, 727-737. [CrossRef]

5. Abdo, A.A.; et al. [Fermi LAT Collaboration] Radio-loud narrow-nine Seyfert 1 galaxies as a new class of gamma-ray active galactic nuclei. Astrophys. J. 2009, 707, L142-L147. [CrossRef]

6. Foschini, L.; et al. [Fermi LAT Collaboration] Fermi/LAT discovery of gamma-ray emission from a relativistic jet in the narrow-line Seyfert 1 quasar PMN J0948+0022. In Accretion and Ejection in AGNs: A Global View; Maraschi, L., Ghisellini, G., Della Ceca, R., Tavecchio, F., Eds.; ASP Conference Series; ASP: San Francisco, CA, USA, 2010; Volume 427, pp. 243-248.

7. Foschini, L.; Ciroi, S.; Berton, M.; Vercellone, S.; Romano, P.; Braito, V. Mapping the Narrow-Line Seyfert 1 Galaxy 1H 0323+342. Universe 2019, 5, 199. [CrossRef]

8. Fan, X.-L. Revisiting the Fraction of Radio-Loud Narrow Line Seyfert 1 Galaxies with LoTSS DR1. Universe 2020, 6, 45. [CrossRef]

9. Vercellone, S.; Foschini, L.; Romano, P.; Böttcher, M.; Boisson, C. High-Energy and Very High-Energy Constraints from Log-Parabolic Spectral Models in Narrow-Line Seyfert 1 Galaxies. Universe 2020, 6, 54. [CrossRef]

10. Liu, X.; Chang, N.; Han, Z.; Wang, X. The Jet-Disk Coupling of Seyfert Galaxies from a Complete Hard X-ray Sample. Universe 2020, 6, 68. [CrossRef]

11. Pan, X.; Zhou, H.; Jiang, P. Obscuration and Scattering in Narrow-Line Seyfert 1s. Universe 2020, 6, 73. [CrossRef]

(C) 2020 by the author. Licensee MDPI, Basel, Switzerland. This article is an open access article distributed under the terms and conditions of the Creative Commons Attribution (CC BY) license (http://creativecommons.org/licenses/by/4.0/). 